\title{
Existence, uniqueness and stability properties of positive equilibria for a class of nonlinear cooperative systems
}

Ugo Abara Precious, Francesco Ticozzi and Claudio Altafini

Book Chapter

N.B.: When citing this work, cite the original article.

Part of: Proceedings of 2015 54th IEEE Conference on Decision and Control (CDC), 2015, pp. 4406-4411. ISBN: 9781479978861, 9781479978847, 9781479978854

DOI: https:// doi.org/ 10.1109/CDC.2015.7402907

Copyright: Institute of Electrical and Electronics Engineers (IEEE)

Available at: Linköping University Institutional Repository (DiVA)

http:// urn.kb.se/ resolve?urn=urn:nbn:se:liu:diva-127607 


\title{
Existence, uniqueness and stability properties of positive equilibria for a class of nonlinear cooperative systems
}

\author{
Precious Ugo Abara and Francesco Ticozzi \\ Dept. of Information Engineering, via Gradenigo 6B \\ University of Padova, 35131, Padova, Italy. \\ Claudio Altafini \\ Division of Automatic Control, Dept. of Electrical Engineering, \\ Linköping University, SE-58183, Linköping, Sweden. \\ email: claudio.altafini@liu.se
}

\begin{abstract}
We provide conditions that guarantee existence, uniqueness and stability of strictly positive equilibria for nonlinear cooperative systems associated to vector fields that are concave or subhomogeneous. This class of positive systems describes well interconnected dynamics that are of key interest for communication, biological, economical and neural network applications. These conditions can be formulated directly in terms of the spectral radius of the Jacobian of the system, and do not require to use constant inputs to move the equilibrium point from the origin to the interior of the positive orthant.
\end{abstract}

\section{INTRODUCTION}

Positive nonlinear systems appear naturally in a number of widely different applications in Engineering, Economics, Biology, Ecology, Physiology etc. [2], [4]. Positivity constraints usually originate from state variables that represent intrinsically positive quantities, such as masses, concentrations, probabilities, etc. For instance, nonlinear positive systems are used to model biochemical reactions [21], gene regulatory networks [16], population dynamics, epidemic processes [17], [13], compartmental systems [10], queueing systems, power control in wireless networks [7], [22], [6], certain types of neural networks [9], [8] and many other systems.

Similarly to the linear case [4], in order to guarantee consistency of a model with the positivity of the state, special constraints on the dynamics must be imposed. One common assumption is that the system is cooperative i.e., that the Jacobian at each point is nonnegative. As such, cooperative nonlinear systems are a special case of monotone systems [20], and inherit from monotonicity a series of desirable properties, like the absence of limit cycles and "generic" convergence to attractors for trajectories that remain bounded. However, cooperativity alone is not enough to characterize other key properties, most notably the existence of a single global (positive) attractor.

In order to guarantee that such an attractor exists, a number of additional modeling hypothesis and tools have been tailored to the numerous applicative contexts for which this type of stable behavior is critical. For example, in wireless networks, most power control algorithms assume that the (nonlinear) "interference functions" are scalable [22] (i.e., subhomogeneous [3], [5]). In a completely different field, the (nonlinear) "activation function" of a Hopfieldtype neural network [9], [8] is often monotone and sigmoidal [8], [23], which means that it lacks inflection points once it is restricted to positive values. In gene regulatory network theory, the cooperative case appears as a special case (all activatory links), and asymptotic stability is achieved making use of saturated monotonicities such as Michaelis-Menten functional forms [16].

It is worth remarking that in all these applications the equilibrium point is normally required to be positive. In fact, the origin is typically not very interesting as an equilibrium: for example, a power control algorithm that converges to zero power is meaningless, and similarly for the other applications. A common trick to move the equilibrium point from the origin to the interior of the positive orthant is to add a non-vanishing positive input, usually a constant, to the system dynamics (a current in a neural network, a noise power in the wireless interference function, a constant mRNA synthesis rate in the gene networks) [15], [18]. This trick is standard (and necessary) in linear positive systems [4], but not strictly necessary in the nonlinear case. As a matter of fact, in some cases the extra constant term seems more motivated by the need of guaranteeing positivity of the equilibrium rather than by a true consistency with the problem setting.

The main scope of this paper is to try to understand what classes of nonlinear cooperative systems may lead to existence, uniqueness and stability of a positive equilibrium point with few constraints on the form and structure of the nonlinearities, and without resorting to the use of additive constants to shift the equilibrium. It is shown in the paper that one such class corresponds to nonlinearities that are both monotone and concave. Concavity appears naturally as a common feature in all examples mentioned above: subhomogeneity of order 1 is a proxy for concavity, sigmoidal functions are concave when restricted to the positive semiaxis, and so are Michaelis-Menten functions. Nonlinear cooperative concave dynamical systems lead to simple 
theorems for existence and uniqueness of fixed points [12], [14] and, when the trajectories they induce do not diverge, also stability analysis of the fixed point is easily shown. What is additionally shown in the paper is that all these properties can be formulated as spectral conditions on the Jacobian of the system. In particular, uniqueness and global attractivity of a positive equilibrium correspond to a bound on the spectral radius of the Jacobian at the origin, plus an additional condition that has to hold inside the positive orthant. When the concave nonlinearities are also bounded, as it is the case for most of the aforementioned examples, the spectral radius of the Jacobian at the origin alone decides all the global dynamical features of the system.

Our results hold essentially unchanged when we replace concave functionals with the broader class of subhomogeneous functionals. This is another class for which stability has been studied mostly at the origin [3], [5], or in presence of constant additive terms [7], [22].

Lastly, although the examples mentioned above can all be framed as interconnected systems [11], [19], the conditions we give in this paper are valid in general. While a very rich literature on stability analysis for interconnected systems is available, the spectral conditions we propose are, to the best of our knowledge, new.

\section{PRELIMINARY MATERIAL}

\section{A. Notation and linear algebra}

Throughout this paper let $\mathbb{R}_{+}^{n}$ denote the positive orthant of $\mathbb{R}^{n}, \operatorname{int}\left(\mathbb{R}_{+}^{n}\right)$ its interior, and $\operatorname{bd}\left(\mathbb{R}_{+}^{n}\right)=\mathbb{R}_{+}^{n} \backslash \operatorname{int}\left(\mathbb{R}_{+}^{n}\right)$. If $x_{1}, x_{2} \in \mathbb{R}^{n}, x_{1} \leqslant x_{2}$ means $x_{1, i} \leqslant x_{2, i} \forall i=1, \ldots, n$, and $x_{1} \neq x_{2}$, while $x_{1}<x_{2}$ means $x_{1, i}<x_{2, i} \forall i=1, \ldots, n$, A matrix $A=\left[a_{i j}\right] \in \mathbb{R}^{n \times n}$ is said nonnegative (in the following indicated $A \geqslant 0$ ) if $a_{i j} \geqslant 0 \forall i, j$, and Metzler if $a_{i j} \geqslant 0 \forall i \neq j$. A is irreducible if $\nexists$ a permutation matrix $\Pi$ that renders it block diagonal:

$$
\Pi^{T} A \Pi=\left[\begin{array}{cc}
A_{11} & A_{12} \\
0 & A_{22}
\end{array}\right]
$$

for nontrivial square matrices $A_{11}, A_{22}$. The spectrum of $A$ is denoted $\Lambda(A)=\left\{\lambda_{1}(A), \ldots, \lambda_{n}(A)\right\}$, where $\lambda_{i}(A)$, $i=1, \ldots, n$, are the eigenvalues of $A$. The spectral radius of $A, \rho(A)$, is the smallest positive real number such that $\rho(A) \geqslant\left|\lambda_{i}(A)\right|, \forall i=1, \ldots, n$.

Theorem 1 (Perron-Frobenius). Let $A \in \mathbb{R}^{n \times n} \geqslant 0$ be irreducible. Then $\rho(A)$ is a real, positive, algebraically simple eigenvalue of $A$, of right (resp. left) eigenvector $v>0$ (resp. $w>0$ ).

\section{B. Concave and subhomogeneous vector fields}

Consider a convex set $\mathcal{W} \subset \mathbb{R}^{n}$. A function $f: \mathcal{W} \rightarrow \mathbb{R}^{n}$ is said non-decreasing in $\mathcal{W}$ if $x_{1} \leqslant x_{2}$ implies $f\left(x_{1}\right) \leqslant$ $f\left(x_{2}\right) \forall x_{1}, x_{2} \in \mathcal{W}$. It is said increasing if in addition $x_{1}<x_{2}$ implies $f\left(x_{1}\right)<f\left(x_{2}\right)$.

Given $\mathcal{W} \subset \mathbb{R}^{n}$ convex, $f: \mathcal{W} \rightarrow \mathbb{R}^{n}$ is said concave if

$$
f\left(\alpha x_{1}+(1-\alpha) x_{2}\right) \geqslant \alpha f\left(x_{1}\right)+(1-\alpha) f\left(x_{2}\right)
$$

$\forall x_{1}, x_{2} \in \mathcal{W}$ and $\forall 0 \leqslant \alpha \leqslant 1$. It is said strictly concave if the inequality in (1) is strict in $0<\alpha<1 \forall x_{1}, x_{2} \in \mathcal{W}$, $x_{1} \neq x_{2}$. For a concave vector field $f$, the tangent vector must always overestimate $f$ at any point and viceversa. Therefore we have that a $C^{1}$ vector field $f: \mathcal{W} \rightarrow \mathbb{R}^{n}$ is concave if and only if

$$
f\left(x_{1}\right) \leqslant f\left(x_{2}\right)+\frac{\partial f\left(x_{2}\right)}{\partial x}\left(x_{1}-x_{2}\right)
$$

$\forall x_{1}, x_{2} \in \mathcal{W} . f$ is strictly concave if (2) holds strictly $\forall x_{1}, x_{2} \in \mathcal{W}, x_{1} \neq x_{2}$. Clearly, $f$ strictly concave and non-decreasing means $f$ increasing.

The vector field $f: \mathcal{W} \rightarrow \mathbb{R}^{n}$ is said subhomogeneous of degree $\tau>0$ if

$$
f(\alpha x) \geqslant \alpha^{\tau} f(x)
$$

$\forall x \in \mathcal{W}$ and $0 \leqslant \alpha \leqslant 1$, and strictly subhomogeneous if the inequality (3) holds strictly $\forall x \in \mathcal{W}$ and $0<\alpha<1$.

In the case of $f(0) \geqslant 0$, concavity is related to subhomogeneity of degree 1 .

Proposition 1 Consider $\mathcal{W} \subset \mathbb{R}^{n}$ convex, $0 \in \mathcal{W}$, and let $f: \mathcal{W} \rightarrow \mathbb{R}^{n}$ be a vector field such that $f(0) \geqslant 0$. If $f(x)$ is concave then $f(x)$ is subhomogeneous of degree 1 .

Proof: Choosing $x_{2}=0$ in (1), we have

$$
f\left(\alpha x_{1}\right) \geqslant \alpha f\left(x_{1}\right)+(1-\alpha) f(0),
$$

or $f\left(\alpha x_{1}\right) \geqslant \alpha f\left(x_{1}\right)$, since $(1-\alpha) f(0) \geqslant 0$.

\section{Positive and cooperative systems}

Given a system

$$
\dot{x}=h(x), \quad x(0)=x_{o}
$$

denote $x\left(t, x_{o}\right)$ its forward solution from the initial condition $x_{o}$ (assumed to be defined $\forall t \in[0, \infty)$ ). The system (4) is said positive if $x\left(t, x_{o}\right) \in \mathbb{R}_{+}^{n} \forall x_{o} \in \mathbb{R}_{+}^{n}$, i.e., $\mathbb{R}_{+}^{n}$ is forward-invariant for (4). Assuming uniqueness of the solution of (4), it is shown for instance in [1] that a necessary and sufficient condition for positivity is that $x_{i}=0$ implies $h_{i}(x) \geqslant 0 \forall x \in \operatorname{bd}\left(\mathbb{R}_{+}^{n}\right)$.

The system (4) is said monotone if $\forall x_{1}, x_{2} \in \mathcal{W}$ with $x_{1} \leqslant x_{2}$ it holds that $x\left(t, x_{1}\right) \leqslant x\left(t, x_{2}\right)$. The Kamke condition gives an easily testable characterization of monotonicity [20] par. 3.1. The vector field $h(x): \mathcal{W} \rightarrow \mathbb{R}^{n}$ is said to be of type-K or to satisfy the Kamke condition if for each $i=1, \ldots, n, h_{i}(a) \leqslant h_{i}(b) \forall a, b \in \mathcal{W}$ satisfying $a \leqslant b$ and $a_{i}=b_{i}$. A type-K system is monotone. For $C^{1}$ vector fields, type-K systems admit an infinitesimal characterization in terms of the signs of the Jacobian. We are particularly interested in a subclass of monotone systems called cooperative systems. A vector field $h: \mathcal{W} \rightarrow \mathbb{R}^{n}$ is said cooperative if the Jacobian matrix $H(x)=\frac{\partial h}{\partial x}$ is Metzler $\forall x \in \mathcal{W}$. Similarly, the system (4) is said cooperative if the vector field $h$ is cooperative on $\mathcal{W}=\mathbb{R}_{+}^{n} \backslash\{0\}$. From $H(x)$ Metzler, it can be easily shown that if the system (4) is cooperative then $\mathbb{R}_{+}^{n}$ is a forward-invariant set for it, i.e., the system (4) is a positive system. 
An important property of cooperative systems that will be used to prove convergence is given by the following lemma whose proof can be found e.g. in [20] Prop. 3.2.1.

Lemma 1 Let $\mathcal{W}$ be open and $h(x): \mathcal{W} \rightarrow \mathbb{R}^{n}$ be a cooperative vector field. Assume $\exists x \in \mathcal{W}$ for which $h(x)<0$ (resp. $h(x)>0$ ). Then the trajectory $x\left(t, x_{o}\right)$ of (4) is decreasing (resp. increasing) for $t \geqslant 0$. In the case $h(x) \leqslant 0$ (resp. $h(x) \geqslant 0)$, the trajectory $x\left(t, x_{o}\right)$ of (4) is non-increasing (resp. non-decreasing).

\section{CONCAVE SYSTEMS: EQUILIBRIA AND STABILITY}

The specific class of nonlinear positive systems considered in this paper is the following:

$$
\dot{x}=\Delta(-x+f(x))
$$

where $x \in \mathbb{R}_{+}^{n}, f: \mathbb{R}_{+}^{n} \rightarrow \mathbb{R}_{+}^{n}$ is a $C^{1}$ cooperative vector field such that $F(x)=\frac{\partial f(x)}{\partial x} \geqslant 0 \forall x \in \mathbb{R}_{+}^{n}$, and $\Delta=\operatorname{diag}\left(\delta_{1}, \ldots, \delta_{n}\right), \delta_{i}>0$. The presence of a negative diagonal term in (5) implies that the complete Jacobian of (5) is Metzler, hence (5) is a cooperative system.

\section{A. Existence and uniqueness of equilibria}

We want to determine conditions on $f$ such that (5) admits a unique positive fixed point:

$$
\exists \text { unique } x^{*} \in \operatorname{int}\left(\mathbb{R}_{+}^{n}\right) \text { such that } f\left(x^{*}\right)=x^{*}
$$

For existence, we can use the following theorem, valid for non-decreasing functions, see [12].

Theorem 2 (Tarski fixed point theorem). Given $\mathcal{W} \subset \mathbb{R}^{n}$ convex, assume $f: \mathcal{W} \rightarrow \mathcal{W}$ is a nondecreasing function such that $f\left(x_{1}\right)>x_{1}$ for some $x_{1} \in \mathcal{W}, x_{1}>0$ and $f\left(x_{2}\right)<$ $x_{2}$ for some $x_{2} \in \mathcal{W}, x_{2}>x_{1}$. Then $\exists x^{*} \in \mathcal{W}$ such that $f\left(x^{*}\right)=x^{*}$.

In the following we shall focus on the case of $\mathcal{W}=\mathbb{R}_{+}^{n}$ i.e., on positive cooperative systems. Under some extra condition like concavity, (5) can be shown to have a unique fixed point. The following result is from Kennan [12].

Theorem 3 Let $f: \mathbb{R}_{+}^{n} \rightarrow \mathbb{R}_{+}^{n}$ be such that

1) $f$ is strictly concave,

2) $f$ is increasing,

3) $f(0) \geqslant 0$,

4) $\exists x_{1} \in \operatorname{int}\left(\mathbb{R}_{+}^{n}\right)$ such that $f\left(x_{1}\right)>x_{1}$,

5) $\exists x_{2} \in \operatorname{int}\left(\mathbb{R}_{+}^{n}\right), x_{2}>x_{1}$, such that $f\left(x_{2}\right)<x_{2}$, then $\exists$ unique $x^{*} \in \operatorname{int}\left(R_{+}^{n}\right)$ such that $x^{*}=f\left(x^{*}\right)$.

\section{B. A spectral characterization of existence and uniqueness of equilibria}

The following theorem gives a spectral condition for the existence and uniqueness of a fixed point, in the case of concave and increasing vector fields.

Theorem 4 Consider the system (5), with $f: \mathbb{R}_{+}^{n} \rightarrow \mathbb{R}_{+}^{n}$ a $C^{1}$, strictly concave and increasing vector field, $f(0)=0$.
Assume $F(x) \geqslant 0$ and irreducible $\forall x \in \mathbb{R}_{+}^{n}$. If the following conditions hold

1) $\rho(F(0))>1$;

2) $\exists x_{2} \in \operatorname{int}\left(\mathbb{R}_{+}^{n}\right)$ such that $\rho\left(F\left(x_{2}\right)\right)<1$.

then the system admits a unique positive equilibrium.

In order to prove this Theorem we will use the following Lemma:

Lemma 2 Let $f: \mathbb{R}_{+}^{n} \rightarrow \mathbb{R}_{+}^{n}$ be a $C^{1}$, strictly concave and increasing vector field, $f(0)=0$. Assume $F(x) \geqslant 0$ and irreducible $\forall x \in \mathbb{R}_{+}^{n}$. Then

1) $\rho(F(0))>1$ if and only if $\exists x_{1} \in \operatorname{int}\left(\mathbb{R}_{+}^{n}\right)$ such that $f\left(x_{1}\right)>x_{1}$.

2) $\exists \bar{x} \in \operatorname{int}\left(\mathbb{R}_{+}^{n}\right)$ such that $\rho(F(\bar{x}))<1$ if and only if $\exists x_{2} \in \operatorname{int}\left(\mathbb{R}_{+}^{n}\right)$, such that $f\left(x_{2}\right)<x_{2}$.

Proof: [Proof of 1] By contradiction, suppose that $\rho(F(0))=\rho_{0} \leqslant 1$ and that $\exists x_{1} \in \mathbb{R}_{+}^{n}, x_{1}>0$ such that $f\left(x_{1}\right)>x_{1}$. Let $w_{0}$ be the left eigenvector of $F(0)$ corresponding to $\rho_{0}$. Since $F(0) \geqslant 0$ and irreducible, from the Perron-Frobenius theorem, $w_{0}>0$. Let $g: \mathbb{R}_{+}^{n} \rightarrow \mathbb{R}^{n}$ be defined as $g(x)=f(x)-x$, of Jacobian $G(x)=-I+F(x)$. From the strict concavity of $f$, also $g$ is strictly concave since it is a linear combination of concave functions. Since $f(x)>x \Longleftrightarrow g(x)>0$, from (2) the following relationship holds

$$
g\left(x_{1}\right)<g(0)+G(0) x_{1}=(-I+F(0)) x_{1} .
$$

Multiplying both sides by $w_{0}^{T}$ the inequality becomes

$$
w_{0}^{T} g\left(x_{1}\right)<-w_{0}^{T} x_{1}+w_{0}^{T} F(0) x_{1}=-w_{0}^{T} x_{1}+\rho_{0} w_{0}^{T} x_{1},
$$

or

$$
w_{0}^{T}\left(g\left(x_{1}\right)+x_{1}-\rho_{0} x_{1}\right)<0 .
$$

Since $w_{0}>0$, and defining $\varepsilon$ as $\varepsilon \triangleq 1-\rho_{0} \geqslant 0$, (6) implies that $\exists i \in\{1, \ldots, n\}$ such that

$$
g_{i}\left(x_{1}\right)+\left(1-\rho_{0}\right) x_{1_{i}}<0 \Longrightarrow g_{i}\left(x_{1}\right)<-\varepsilon x_{1_{i}} \leqslant 0,
$$

which is a contradiction, since our hypothesis implies $x_{1_{i}}>$ 0 and $g_{i}\left(x_{1}\right)>0$ for all $i$.

Now suppose $\rho(F(0))=\rho_{0}>1$. We want to show that there exists $x_{1}>0$ such that $f\left(x_{1}\right)>x_{1}$. From Taylor's theorem and from the $C^{1}$ assumption for $f$, we have that for every $i=1, \ldots, n$ the following holds $\forall x_{0} \in \mathbb{R}_{+}^{n}$

$$
f_{i}(x)=f_{i}\left(x_{0}\right)+\frac{\partial f_{i}\left(x_{0}\right)}{\partial x}\left(x-x_{0}\right)+\phi_{i}\left(x-x_{0}\right),
$$

where $\phi_{i}: \mathbb{R}^{n} \rightarrow \mathbb{R}$ is such that

$$
\lim _{x \rightarrow x_{0}} \frac{\phi_{i}\left(x-x_{0}\right)}{\left\|x-x_{0}\right\|}=0 .
$$

Joining all $i=1, \ldots, n$ equations, yields

$$
f(x)=f\left(x_{0}\right)+F\left(x_{0}\right)\left(x-x_{0}\right)+\phi\left(x-x_{0}\right) .
$$

Let $v_{0}>0$ be the right eigenvector corresponding to the Perron-Frobenius eigenvalue $\rho(F(0))=\rho_{0}>1$. From 
Taylor's approximation in (7) and by choosing $x_{0} \equiv 0$ and $x \equiv x_{1}$, we have

$$
f\left(x_{1}\right)=f(0)+F(0) x_{1}+\phi\left(x_{1}\right) .
$$

Since we are interested in finding a vector $x_{1}>0$ such that $f\left(x_{1}\right)>x_{1}$, let us choose $x_{1}=\gamma v_{0}, \gamma>0$. The vector $x_{1}$ is clearly a positive vector and

$$
\lim _{\gamma \rightarrow 0} \frac{\phi\left(x_{1}\right)}{\gamma}=0
$$

With these choices, equation (8) becomes

$$
f\left(x_{1}\right)=\rho(F(0)) x_{1}+\phi\left(x_{1}\right)=\rho_{0} x_{1}+\phi\left(x_{1}\right)
$$

or, rewriting $\rho_{0}$ as $\rho_{0}=1+\varepsilon, \varepsilon>0$,

$$
f\left(x_{1}\right)=x_{1}+\varepsilon x_{1}+\phi\left(x_{1}\right) \text {. }
$$

Recalling that $x_{1}=\gamma v_{0}$, it is

$$
f\left(x_{1}\right)=x_{1}+\gamma\left(\varepsilon v_{0}+\frac{1}{\gamma} \phi\left(x_{1}\right)\right)
$$

and, from (9), $\varepsilon v_{0}+(1 / \hat{\gamma}) \phi\left(x_{1}\right)>0$ for an appropriate small $\hat{\gamma} \neq 0$, or

$$
f\left(x_{1}\right)=x_{1}+\hat{\gamma}(\text { something positive })>x_{1},
$$

which completes the proof of this first part.

[Proof of 2] Suppose there $\exists \bar{x} \in \mathbb{R}_{+}^{n}$ such that $\rho(F(\bar{x}))=$ $\bar{\rho}<1$. We first show that there exists $x_{2} \in \mathbb{R}_{+}^{n}$, such that $f\left(x_{2}\right)<x_{2}$. Consider again $g(x)=f(x)-x$. We assume $\exists i \in\{1, \ldots, n\}$ such that $g_{i}(\bar{x}) \geqslant 0$, otherwise the proof would be finished since $g(\bar{x})<0$ implies $f(\bar{x})<\bar{x}$. Since $F(\bar{x}) \geqslant 0$ and irreducible, from the Perron-Frobenius theorem we have that $\bar{\rho}$ is a real positive eigenvalue of $F(\bar{x})$. The Jacobian matrix of $g$ in $\bar{x}$ is $G(\bar{x})=-I+F(\bar{x})$ and we can easily derive its eigenvalue with maximum real part to be

$$
\begin{aligned}
-\varepsilon & \triangleq \max \{\operatorname{Re}(\lambda), \lambda \in \Lambda(G(\bar{x}))\} \\
& =\max \{\operatorname{Re}(\lambda), \lambda \in \Lambda(-I+F(\bar{x}))\} \\
& =-1+\max \{\operatorname{Re}(\lambda), \lambda \in \Lambda(F(\bar{x}))\} \\
& =-1+\bar{\rho}
\end{aligned}
$$

where $\varepsilon>0$ since $\bar{\rho}<1$. From the Perron-Frobenius theorem, the right eigenvector of $F(\bar{x})$ relative to $\bar{\rho}$, call it $v$, must be positive, $v>0 . v$ is also the right eigenvector of $G(\bar{x})$ relative to $-\varepsilon$ :

$$
G(\bar{x}) v=(-I+F(\bar{x})) v=(-1+\bar{\rho}) v=-\varepsilon v .
$$

We need to show that there exists $x_{2}$ such that $g\left(x_{2}\right)<0$. Let us define $x_{2}$ as

$$
x_{2}=\bar{x}+\gamma v \quad \gamma \in \mathbb{R}_{+}, \gamma>0 .
$$

As $v>0$ and $\gamma$ is positive, it is clear that $x_{2}>\bar{x}$. The vector field $g$ is strictly concave. Then, from (2), one gets

$$
g\left(x_{2}\right)<g(\bar{x})+G(\bar{x})\left(x_{2}-\bar{x}\right) .
$$

From (11) and (10), the previous expression becomes

$$
g\left(x_{2}\right)<g(\bar{x})-\varepsilon \gamma v .
$$

By choosing an appropriate $\gamma$ the right hand side can be made negative. For example

$$
\gamma \triangleq \frac{1}{\varepsilon} \frac{\max _{i=1, \ldots, n}\left\{g_{i}(\bar{x})\right\}}{\min _{j=1, \ldots, n}\left\{v_{j}\right\}}
$$

implies $\left(\mathbf{1}=\left[\begin{array}{lll}1 & \ldots & 1\end{array}\right]^{T}\right)$

$$
\begin{aligned}
g\left(x_{2}\right) & <g(\bar{x})-\frac{\max _{i=1, \ldots, n}\left\{g_{i}(\bar{x})\right\}}{\min _{j=1, \ldots, n}\left\{v_{j}\right\}} v \\
& \leqslant g(\bar{x})-\max _{i=1, \ldots, n}\left\{g_{i}(\bar{x})\right\} \mathbf{1} \leqslant 0
\end{aligned}
$$

since $\left(v_{i} / \min _{j=1, \ldots, n}\left\{v_{j}\right\}\right) \geqslant 1, \forall i$. The proof of sufficiency is completed since $g\left(x_{2}\right)<0 \Longrightarrow f\left(x_{2}\right)<x_{2}$. To show the necessity part, assume $\exists x_{2} \in \mathbb{R}_{+}^{n}$, such that $f\left(x_{2}\right)<x_{2}$. To prove this part it is enough to choose $\bar{x}=x_{2}$. Assume by contradiction that $\rho\left(F\left(x_{2}\right)\right) \geqslant 1$. Then by strict concavity of $f$ the following holds

$$
f(0)<f\left(x_{2}\right)+F\left(x_{2}\right)\left(0-x_{2}\right)
$$

which, from $f(0)=0$ and $f\left(x_{2}\right)<x_{2}$, yields

$$
0<x_{2}-F\left(x_{2}\right) x_{2} .
$$

Let $w>0$ be the left Perron-Frobenius eigenvector corresponding to $\rho\left(F\left(x_{2}\right)\right)$. Multiplying both sides of (12) and rearranging

$$
w^{T} F\left(x_{2}\right) x_{2}=\rho\left(F\left(x_{2}\right)\right) w^{T} x_{2}<w^{T} x_{2}
$$

which is clearly a contradiction if $\rho\left(F\left(x_{2}\right)\right) \geqslant 1$.

Proof: [Proof of Theorem 4] Using Lemma 2 the two conditions of the Theorem become:

1) $\exists x_{1} \in \operatorname{int}\left(\mathbb{R}_{+}^{n}\right)$ such that $f\left(x_{1}\right)>x_{1}$;

2) $\exists x_{2} \in \operatorname{int}\left(\mathbb{R}_{+}^{n}\right)$ such that $f\left(x_{2}\right)<x_{2}$.

When condition 1 holds, $x_{2}$ can always be chosen such that $x_{2}>x_{1}$ (since $x_{1} \in \operatorname{int}\left(\mathbb{R}_{+}^{n}\right)$ can be chosen arbitrary close to 0 ). Hence Theorem 3 applies and the system (5) admits a unique positive equilibrium.

The asymmetry in the proof of the second part of Lemma 2 (in one direction $\bar{x}<x_{2}$, in the other $\bar{x}=x_{2}$ ), can be explained by the value of spectral radius at the equilibrium point, a result itself of independent interest.

Proposition 2 Let $f: \mathbb{R}_{+}^{n} \rightarrow \mathbb{R}_{+}^{n}$ be a strictly concave and increasing vector field, $f(0)=0$. Assume $F(x) \geqslant 0$ and irreducible $\forall x \in \mathbb{R}_{+}^{n}$. If $\exists x^{*} \in \operatorname{int}\left(\mathbb{R}_{+}^{n}\right)$ such that $f\left(x^{*}\right)=x^{*}$, then $\rho\left(F\left(x^{*}\right)\right)<1$.

Proof: The proof is identical to the necessity part of the second condition of Lemma 2, provided one replaces " $f\left(x_{2}\right)<x_{2}$ "with " $f\left(x^{*}\right)=x^{*}$.

Also the following condition is of independent interest.

Proposition 3 Let $f: \mathbb{R}_{+}^{n} \rightarrow \mathbb{R}_{+}^{n}$ be $C^{1}$ strictly concave and increasing. If $\exists x_{2} \in \operatorname{int}\left(\mathbb{R}_{+}^{n}\right)$ such that $f\left(x_{2}\right)<x_{2}$, then $f(x)<x \forall x>x_{2}$. 
Proof: Letting $g(x)=f(x)-x$, we need to show that if $g\left(x_{2}\right)<0$ then $g(x)<0 \forall x>x_{2}$. By contradiction, let us suppose that there exists $k \in\{1, \ldots, n\}$ such that

$$
g_{k}(x) \geqslant 0 \text {. }
$$

Since $g_{k}$ is strictly concave, the upper contour set $S_{\alpha}=\left\{x \in \mathbb{R}_{+}^{n}: g_{k}(x) \geqslant \alpha\right\}$ must be convex for all $\alpha \in \mathbb{R}$. Choosing for example $\alpha=g_{k}\left(x_{2}\right) / 2$, it is clear that $x_{2} \notin S_{\alpha}$ while $x \in S_{\alpha}$. Let us define $\bar{z}$ as $\bar{z}=\bar{\lambda} x+(1-\bar{\lambda}) x_{2}$, where $\bar{\lambda}$ is the smallest real number in $(0,1)$ such that $\bar{z} \in S_{\alpha}$. Clearly $\alpha<0$, thus $0 \in S_{\alpha}$. Then, from the strict concavity of $g_{k}$, the convex combination of 0 and $\bar{z}$ should lie in $S_{\alpha}$, but $g(\beta \bar{z})<\alpha$ for some $\beta<1$. This shows that $S_{\alpha}$ has holes, i.e. it is not convex. Thus it must be $g_{k}(x)<0$ i.e. $f(x)<x \forall x>x_{2}$.

\section{A spectral characterization of stability}

Also the stability character of the equilibrium can be expressed as a spectral condition on the Jacobian.

Theorem 5 Consider the system (5), with $f: \mathbb{R}_{+}^{n} \rightarrow \mathbb{R}_{+}^{n}$ a $C^{1}$, strictly concave and increasing vector field, $f(0)=0$. Assume $F(x) \geqslant 0$ and irreducible $\forall x \in \mathbb{R}_{+}^{n}$. Then

1) the origin is an asymptotically stable equilibrium point for (5) if and only if $\rho(F(0))<1$. In this case the domain of attraction is $\mathcal{A}(0) \supset \mathbb{R}_{+}^{n}$.

2) If $\rho(F(0))>1$ and $\exists x_{2} \in \operatorname{int}\left(\mathbb{R}_{+}^{n}\right)$ such that $\rho\left(F\left(x_{2}\right)\right)<1$, the unique positive equilibrium $x^{*} \in$ $\operatorname{int}\left(\mathbb{R}_{+}^{n}\right)$ of system (5) is asymptotically stable and has domain of attraction $\mathcal{A}\left(x^{*}\right) \supset \mathbb{R}_{+}^{n} \backslash\{0\}$.

Proof: [Proof of 1] From the Perron-Frobenius theorem and from the irreducibility of $F(0), w_{0}$, the left eigenvector of $F(0)$ relative to $\rho_{0}=\rho(F(0))$ is $w_{0}>0$. For the system (5), the diagonal matrix $\Delta$ is positive definite which implies that $\Delta^{-1}$ is positive definite. Let $V: \mathbb{R}_{+}^{n} \rightarrow \mathbb{R}_{+}$be the following Lyapunov function

$$
V(x)=\frac{1}{2} x^{T}\left(\Delta^{-1} w_{0} w_{0}^{T} \Delta^{-1}\right) x .
$$

The matrix $\left(\Delta^{-1} w_{0} w_{0}^{T} \Delta^{-1}\right)$ is clearly symmetric and strictly positive, hence $V(x)>0 \forall x \neq 0, V(0)=0$. Differenting $V$ we have

$$
\begin{aligned}
\dot{V}(x) & =x^{T} \Delta^{-1} w_{0} w_{0}^{T} \Delta^{-1} \dot{x} \\
& =x^{T} \Delta^{-1} w_{0} w_{0}^{T}(-x+f(x)) .
\end{aligned}
$$

From strict concavity of $f$ and from (2) (with $x_{1}=x$ and $x_{2}=0$ ), we have

$$
f(x)<F(0) x
$$

or, multiplying both sides by $w_{0}^{T}$,

$$
w_{0}^{T} f(x)<\rho(F(0)) w_{0}^{T} x=\rho_{0} w_{0}^{T} x .
$$

The assumption $\rho_{0}<1$ gives

$$
w_{0}^{T} f(x)<w_{0}^{T} x
$$

hence in (14) we have

$$
\begin{aligned}
\dot{V} & =-x^{T} \Delta^{-1} w_{0} w_{0}^{T} x+x^{T} \Delta^{-1} w_{0} w_{0}^{T} f(x) \\
& =x^{T} \Delta^{-1} w_{0}\left(-w_{0}^{T} x+w_{0}^{T} f(x)\right) .
\end{aligned}
$$

From condition (15), $-w_{0}^{T} x+w_{0}^{T} f(x)<0$, which implies that $\dot{V}<0$ for all $x \in \mathbb{R}_{+}^{n} \backslash\{0\}$, since $x^{T} \Delta^{-1} w_{0}>0$. The proof holds globally in $\mathbb{R}_{+}^{n}$ since $V$ is radially unbounded. [Proof of 2] Under condition 2, existence and uniqueness of the equilibrium $x^{*} \in \operatorname{int}\left(\mathbb{R}_{+}^{n}\right)$ follow from Theorem 4 . Given $x^{*}$, split $\mathbb{R}_{+}^{n}$ into the regions

$$
\begin{aligned}
& \Omega_{1}=\left\{x \in \mathbb{R}_{+}^{n} \text { such that } x \leqslant x^{*}\right\} \\
& \Omega_{2}=\left\{x \in \mathbb{R}_{+}^{n} \text { such that } x \geqslant x^{*}\right\} \\
& \Omega_{3}=\mathbb{R}_{+}^{n} \backslash\left(\Omega_{1} \cup \Omega_{2}\right) .
\end{aligned}
$$

From cooperativity (and Lemma 1 ) we have that $\Omega_{1}$ and $\Omega_{2}$ are forward invariant. In fact, the monotonicity property

$$
x_{0} \leqslant y_{0} \Longrightarrow x\left(t, x_{0}\right) \leqslant x\left(t, y_{0}\right) \quad \forall t \geqslant 0
$$

implies that on $\Omega_{1}$ we have

$$
x_{0} \leqslant x^{*} \Longrightarrow x\left(t, x_{0}\right) \leqslant x\left(t, x^{*}\right)=x^{*} \quad \forall t \geqslant 0
$$

and on $\Omega_{2}$

$$
x_{0} \geqslant x^{*} \Longrightarrow x\left(t, x_{0}\right) \geqslant x\left(t, x^{*}\right)=x^{*} \quad \forall t \geqslant 0 .
$$

Consider the Lyapunov function

$$
V(x)=\frac{1}{2}\left(x-x^{*}\right)^{T} \Delta^{-1}\left(x-x^{*}\right) .
$$

Clearly $V(x)>0 \forall x \in \mathbb{R}_{+}^{n} \backslash\left\{x^{*}\right\}, V\left(x^{*}\right)=0$. If $g=$ $-x+f(x)$, then differentiating $V(x)$,

$$
\begin{aligned}
\dot{V}(x) & =\left(x-x^{*}\right)^{T} \Delta^{-1} \dot{x}=\left(x-x^{*}\right)^{T} g(x) \\
& =\sum_{i=1}^{n}\left(x_{i}-x_{i}^{*}\right) g_{i}(x) .
\end{aligned}
$$

If $x \in \Omega_{1} \backslash\{0\}, x \neq x^{*}$ then from strict concavity of $f$ (and of $g$ ) and from the uniqueness of the equilibrium, it follows that $g(x) \geqslant 0$ and $\exists i$ such that $g_{i}(x)>0$. This implies that in (16)

$\left(x_{i}-x_{i}^{*}\right) g_{i}(x) \leqslant 0 \forall i$ and $\exists i$ such that $\left(x_{i}-x_{i}^{*}\right) g_{i}(x)<0$.

Similarly, for $x \in \Omega_{2}, x \neq x^{*}$, one has $g(x) \leqslant 0$ and $g_{i}(x)<$ 0 for some $i$, hence (17) holds also in this case. Putting together:

$$
\dot{V}(x)<0 \quad \forall x \in \Omega_{1} \cup \Omega_{2} \backslash\{0\} .
$$

As for $\Omega_{3}$, if $x_{0} \in \Omega_{3}$ then $\exists$ two positive real constants $\alpha<1$ and $\beta>1$ such that $\alpha x_{0} \in \Omega_{1}$ and $\beta x_{0} \in \Omega_{2}$. It then follows from invariance of $\Omega_{1}$ and $\Omega_{2}$ that $\forall t \geqslant 0$

$$
\alpha x_{0} \leqslant x_{0} \leqslant \beta x_{0} \Longrightarrow x\left(t, \alpha x_{0}\right) \leqslant x\left(t, x_{0}\right) \leqslant x\left(t, \beta x_{0}\right) \text {. }
$$

Since we already know that $x\left(t, \alpha x_{0}\right) \rightarrow x^{*}$, and that $x\left(t, \beta x_{0}\right) \rightarrow x^{*}$, it must be $x\left(t, x_{0}\right) \rightarrow x^{*}$, i.e., $x^{*}$ is asymptotically stable. Since $V(x)$ is radially unbounded, convergence to $x^{*}$ is global in $\mathbb{R}_{+}^{n} \backslash\{0\}$. 
D. A special case: spectral conditions that depend on the degradation rates

If in place of (5), we consider the system

$$
\dot{x}=-\Delta x+\tilde{f}(x)
$$

then the results of Theorems 4 and 5 depend explicitly on the values of the degradation rate constants $\delta_{i}$. Since $\Delta$ invertible, (18) can be rewritten as

$$
\dot{x}(t)=\Delta\left(-x(t)+\Delta^{-1} \tilde{f}(x(t))\right),
$$

and defining $f$ as $f=\Delta^{-1} \tilde{f}(x(t))$, we can readily apply Theorems 4 and 5 to it. However, the sufficient conditions can be made sharper in terms of the $\delta_{i}$ as in the following theorem (presented without proof for lack of space).

Theorem 6 Consider the system (18), with $\tilde{f}: \mathbb{R}_{+}^{n} \rightarrow \mathbb{R}_{+}^{n}$ $a C^{1}$, strictly concave and increasing vector field, $\tilde{f}(0)=0$. Assume $\tilde{F}(x)=\frac{\partial \tilde{f}(x)}{\partial x}$ irreducible $\forall x \in \mathbb{R}_{+}^{n}$. Then

1) the origin is an asymptotically stable equilibrium point for (18) if and only if $\rho(\tilde{F}(0))<\delta_{\min }=\min \left(\delta_{i}\right)$. In this case the domain of attraction is $\mathcal{A}(0) \supset \mathbb{R}_{+}^{n}$.

2) If $\rho(\tilde{F}(0))>\delta_{\max }=\max \left(\delta_{i}\right)$ and $\exists x_{2} \in \operatorname{int}\left(\mathbb{R}_{+}^{n}\right)$ such that $\rho\left(\tilde{F}\left(x_{2}\right)\right)<\delta_{\min }$, the system (18) admits a unique positive equilibrium $x^{*} \in \operatorname{int}\left(\mathbb{R}_{+}^{n}\right)$ which is asymptotically stable and has domain of attraction $\mathcal{A}\left(x^{*}\right) \supset \mathbb{R}_{+}^{n} \backslash\{0\}$.

\section{E. Generalization to subhomogeneous vector fields}

The results of Theorem 4 and Theorem 5 can be extended to subhomogeneous vector fields.

Theorem 7 Consider the system (5) with $f: \mathbb{R}_{+}^{n} \rightarrow \mathbb{R}_{+}^{n}$ a $C^{1}$ vector field which is strictly subhomogeneous of degree $0<\tau \leqslant 1$ and increasing. Assume $f(0)=0$ and $F(x) \geqslant 0$ irreducible $\forall x \in \mathbb{R}_{+}^{n}$. If the following conditions hold:

1) $\rho(F(0))>1$;

2) $\exists x_{2} \in \operatorname{int}\left(\mathbb{R}_{+}^{n}\right)$ such that $\rho\left(F\left(x_{2}\right)\right)=\zeta<1$ and $\rho(F(x)) \leqslant \zeta \forall x>x_{2}$,

then the system (5) admits a unique positive equilibrium point $x^{*} \in \operatorname{int}\left(\mathbb{R}_{+}^{n}\right)$ which is asymptotically stable with domain of attraction $\mathcal{A}\left(x^{*}\right) \supset \mathbb{R}_{+}^{n} \backslash\{0\}$.

Also in this case we have to omit the proof for lack of space.

\section{APPLICATION TO INTERCONNECTED SYSTEMS}

In this section we consider a system on a given graph $\mathcal{G}$, in which the state of a node propagates to its first neighbors following the direction of the edges. The incoming interactions at a node obey a principle of linear superposition of the effects. In addition, the network includes first order degradation terms $\delta_{i} i=1, \ldots, n$, on the diagonal. We assume that a node $j$ exerts the same form of influence on all its neighbors, up to a scaling constant which corresponds to the weight of the edge connecting $j$ with $i$. If $A=\left[a_{i j}\right] \geqslant 0$ is the weighted adjacency matrix of the network, and $\psi_{j}\left(x_{j}\right)$ is the functional form of the interaction from node $j$ to all its neighbors, then we can write the system (18) as

$$
\frac{d x}{d t}=A \psi(x)-\Delta x
$$

where $\psi(x)=\left[\psi_{1}\left(x_{1}\right) \ldots \psi_{n}\left(x_{n}\right)\right]^{T}$. We assume that $\psi_{j}\left(x_{j}\right)$ is monotone and strictly concave. Additionally, we enforce a boundedness condition on $\psi_{j}$ :

$$
\lim _{x_{j} \rightarrow+\infty} \psi_{j}\left(x_{j}\right)=1 \text {. }
$$

While not necessary to apply Theorem 4 and Theorem 6 , the condition (20) implies that the existence of $x_{2}>0$ such that $\rho\left(F\left(x_{2}\right)\right)<1$ in these two theorems is automatically satisfied. The system (19) with this extra hypothesis resembles closely a cooperative additive neural network of Hopfield type but without external inputs. Such neural networks models are sometimes referred to as (cooperative) CohenGrossberg neural networks [23]. An example of $\psi_{j}\left(x_{j}\right)$ monotone, strictly concave and saturating is given by a socalled Boltzmann sigmoid (or shifted logistic) [8]

$$
\psi_{j}\left(x_{j}\right)=\frac{1-e^{-\frac{x_{j}}{\theta_{j}}}}{1+e^{-\frac{x_{j}}{\theta_{j}}}} .
$$

For (21), $0 \leqslant \psi_{j}\left(x_{j}\right) \leqslant 1$ when $x_{j} \geqslant 0$, and

$$
\frac{\partial \psi_{j}}{\partial x_{j}}=\frac{1}{2 \theta_{j}}\left(1+\psi_{j}\left(x_{j}\right)\right)\left(1-\psi_{j}\left(x_{j}\right)\right)>0 \quad \forall x \geqslant 0 .
$$

Since $\psi_{j}(0)=0$, the Jacobian linearization at the origin is $1 /\left(2 \theta_{j}\right)$. In particular, when $x_{j} \ll \theta_{j}$ then $\psi_{j}\left(x_{j}\right) \simeq x_{j} / \theta_{j}$ is a first order rate law, while when $x_{j} \gg \theta_{j}$ then $\psi_{i j}\left(x_{j}\right) \simeq 1$ behaves like a zero order rate law. Many other monotone concave nonlinearities can be used in place of (21). One example is the following "Michaelis-Menten" functional, mutuated from biochemical reaction theory:

$$
\psi_{j}\left(x_{j}\right)=\frac{x_{j}}{\theta_{j}+x_{j}}
$$

where in this case the slope at $x_{j}=0$ is $\frac{1}{\theta_{j}}$ See Fig. 1 for a comparison of the different $\psi_{j}\left(x_{j}\right)$ just introduced. Others can be found in the neural network literature [8], [11]. For

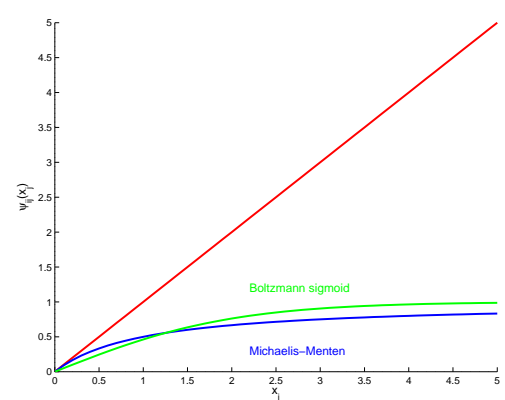

Fig. 1. Monotone concave sigmoidals.

(19), denote $\tilde{F}(x)=A \frac{\partial \psi(x)}{\partial x}$ the Jacobian linearization of $\tilde{f}(x)=A \psi(x)$. From (22) and $A \geqslant 0$, it follows that $\tilde{F}(x) \geqslant$ 
$0 \forall x \in \mathbb{R}_{+}^{n}$. This implies that (19) is a positive cooperative system.

Calling $\mathcal{G}(\tilde{F}(x))$ the graph whose adjacency matrix is $\tilde{F}(x), \tilde{F}(x)$ and $A$ have the same graph at each point of $\mathbb{R}^{n}$, hence irreducibility of $A$ implies irreducibility of $\mathcal{G}(\tilde{F}(x))$ $\forall x \in \operatorname{int}\left(\mathbb{R}_{+}^{n}\right)$. Since $\psi_{j}\left(x_{j}\right)$ is strictly concave, so is $\tilde{F}(x)$. Hence Theorem 6 is applicable.

Example 1 For $n=2$ agents, assuming for example

$$
A=\left[\begin{array}{ll}
0 & 1 \\
1 & 0
\end{array}\right]
$$

the system (19) with the functions (21) becomes

$$
\left\{\begin{array}{l}
\frac{d x_{1}}{d t}=\frac{1-e^{-\frac{x_{2}}{\theta_{2}}}}{1+e^{-\frac{x_{2}}{\theta_{2}}}}-\delta_{1} x_{1} \\
\frac{d x_{2}}{d t}=\frac{1-e^{-\frac{x_{1}}{\theta_{1}}}}{1+e^{-\frac{x_{1}}{\theta_{1}}}}-\delta_{2} x_{2}
\end{array}\right.
$$

In this case, it is possible to use phase plane analysis to verify the conditions of Theorem 6 analytically. The nullclines of this system are given by

$$
\left\{\begin{array}{l}
x_{1, \text { null }}=\frac{1-e^{-\frac{x_{2}}{\theta_{2}}}}{\delta_{1}\left(1+e^{-\frac{x_{2}}{\theta_{2}}}\right)} \\
x_{2, \text { null }}=\frac{1-e^{-\frac{x_{1}}{\theta_{1}}}}{\delta_{2}\left(1+e^{-\frac{x_{1}}{\theta_{1}}}\right)}
\end{array}\right.
$$

which for positive $\delta_{i}$ and $\theta_{i}$ have at most 2 intersections in $\mathbb{R}_{+}^{2}$, see Fig. 2. If we look at the graphs of (24), then the slopes at $x=0$ are given by the lines

$$
\left\{\begin{array}{l}
x_{2}=2 \theta_{2} \delta_{1} x_{1} \\
x_{2}=\frac{1}{2 \theta_{1} \delta_{2}} x_{1}
\end{array}\right.
$$

We have therefore a bifurcation at $\delta_{1} \delta_{2}=\frac{1}{4 \theta_{1} \theta_{2}}$ :

- when $\delta_{1} \delta_{2}>\frac{1}{4 \theta_{1} \theta_{2}}$ the $x_{1}$-nullcline and the $x_{2}$-nullcline intersect only in one equilibrium $\left(x_{0}^{*}=0\right)$;

- when $\delta_{1} \delta_{2}<\frac{1}{4 \theta_{1} \theta_{2}}$ the $x_{1}$-nullcline and the $x_{2}$-nullcline intersect in 2 equilibria: $x_{0}^{*}=0, x_{1}^{*}>0$.

See Fig. 2 for an example. The Jacobian of the interaction
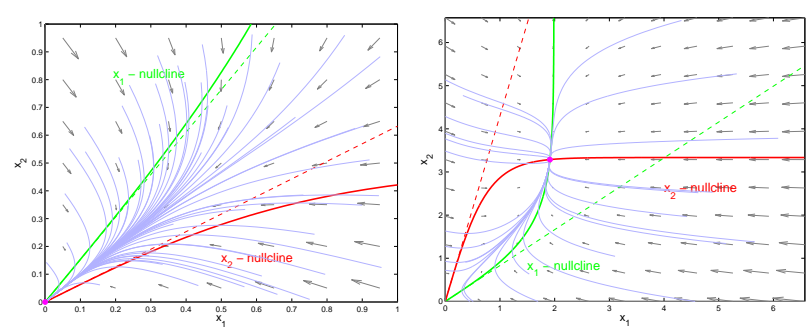

Fig. 2. Example 1. Nullclines are shown as solid lines, slopes at $x=0$ are shown as dashed lines, and a few trajectories are shown in blue solid lines. Left panel: $x_{0}^{*}=0$ is the only equilibrium point. Right panel: $x_{0}^{*}=$ 0 (unstable) and $x_{1}^{*}>0$ (asymptotically stable) are the two equilibrium points.

part alone (omitting the argument in $\psi_{i}$ )

$$
\tilde{F}(x)=\left[\begin{array}{cc}
0 & \frac{\left(1+\psi_{2}\right)\left(1-\psi_{2}\right)}{2 \theta_{2}} \\
\frac{\left(1+\psi_{1}\right)\left(1-\psi_{1}\right)}{2 \theta_{1}} & 0
\end{array}\right]
$$

has eigenvalues

$$
\lambda_{1,2}^{\text {interact. }}= \pm \sqrt{\frac{\left(1+\psi_{1}\right)\left(1-\psi_{1}\right)\left(1+\psi_{2}\right)\left(1-\psi_{2}\right)}{4 \theta_{1} \theta_{2}}}
$$

which implies that the spectral radius of the interaction part is

$$
\rho(\tilde{F}(x))=\sqrt{\frac{\left(1+\psi_{1}\right)\left(1-\psi_{1}\right)\left(1+\psi_{2}\right)\left(1-\psi_{2}\right)}{4 \theta_{1} \theta_{2}}}
$$

from which

$$
\rho(\tilde{F}(0))>\rho(\tilde{F}(x)) \quad \forall x \neq 0,
$$

see Fig. 3. The Jacobian of the entire system (23) is $\tilde{F}(x)-\Delta$ and its eigenvalues are solutions of

$\lambda_{1,2}=\frac{\left(\delta_{1}+\delta_{2}\right) \pm \sqrt{\left(\delta_{1}-\delta_{2}\right)^{2}+\frac{\left(1+\psi_{1}\right)\left(1-\psi_{1}\right)\left(1+\psi_{2}\right)\left(1-\psi_{2}\right)}{\theta_{1} \theta_{2}}}}{2}$

Considering an equilibrium point of (23), the conditions for

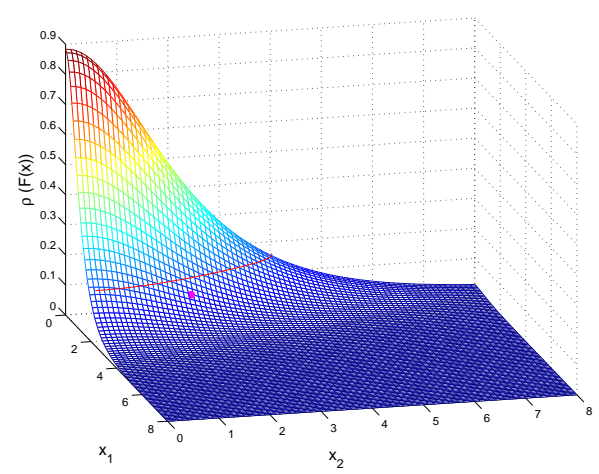

Fig. 3. Example 1. The spectral radius $\rho(\tilde{F}(x))$ is nonnegative and decreasing with $x$. The red contour represents the bifurcation curve $\delta_{1} \delta_{2}=$ $\frac{1}{4 \theta_{1} \theta_{2}}$. The magenta dot represents $x_{1}^{*}$.

its stability are

$$
\begin{aligned}
\operatorname{tr}(\tilde{F}(x)-\Delta) & =-\left(\delta_{1}+\delta_{2}\right)<0 \\
\operatorname{det}(\tilde{F}(x)-\Delta) & =\delta_{1} \delta_{2}-\rho^{2}(\tilde{F}(x))>0 .
\end{aligned}
$$

For example in $x_{0}^{*}=0$, the second condition becomes

$$
\delta_{1} \delta_{2}>\rho^{2}(\tilde{F}(0))=\frac{1}{4 \theta_{1} \theta_{2}}
$$

i.e., when $x_{0}^{*}=0$ is the only equilibrium point of (23) then it must be asymptotically stable. When instead $\delta_{1} \delta_{2}<\frac{1}{4 \theta_{1} \theta_{2}}$ then $x_{0}^{*}$ becomes a saddle point. Since $\left(1+\psi_{i}\right)\left(1-\psi_{i}\right)$ is monotonically decreasing with $x_{i}$, so is $\rho(\tilde{F}(x))$ as a function of $x$, see Fig. 3, and in particular $\lim _{x \rightarrow \infty} \rho(\tilde{F}(x))=0$. Hence when $\delta_{1} \delta_{2}<\frac{1}{4 \theta_{1} \theta_{2}}=\rho^{2}(\tilde{F}(0))$ it must be $\delta_{1} \delta_{2}>$ $\rho^{2}(\tilde{F}(x))$ for $x$ sufficiently large. In particular this must happen on $x_{1}^{*}$, i.e., the positive equilibrium point of (23) must be asymptotically stable whenever it exists. In conclusion, the system (23) experiences a saddle-node bifurcation at $\delta_{1} \delta_{2}=\rho^{2}(\tilde{F}(0))$. If $\Delta$ is given, only the spectral radius 
at 0 is needed to discriminate between the two situations described in Theorem 6.

Example 2 For $n=100$, the two different behaviors predicted by Theorem 6 can be observed, see Fig. 4 .
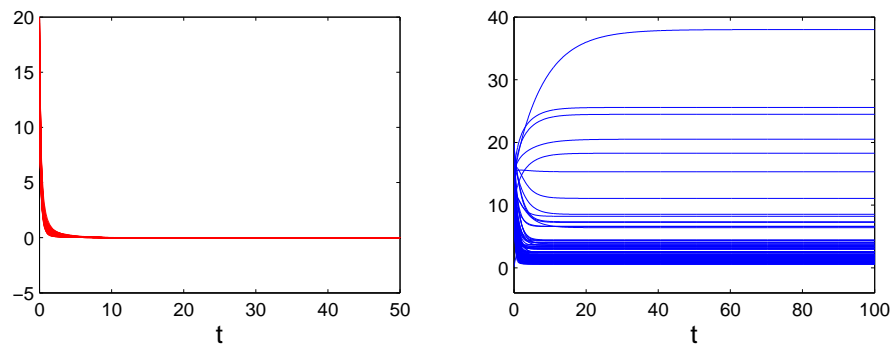

Fig. 4. Example 2. Simulation for a system (19) of $n=100$ nodes, using the functional forms (21) for $\psi$. Left: the origin is asymptotically stable (case 1 of Theorem 6). Right: $x^{*}>0$ is asymptotically stable (case 2 of Theorem 6).

\section{CONCLUSION}

A feature often used in the stability analysis of nonlinear (interconnected) systems is that the nonlinearities are monotone and "declining", meaning, depending on the context, bounded or unbounded sigmoidal, or saturated and without inflection points, or scalable. For positive systems, a generalization of this feature is in terms of monotone and concave vector fields. For them, existence, uniqueness and stability of the (nontrivial) equilibrium point can be investigated efficiently, and reformulated as spectral conditions on the Jacobian of the system.

\section{REFERENCES}

[1] Dirk Aeyels and Patrick De Leenheer. Extension of the PerronFrobenius theorem to homogeneous systems. SIAM J. Control Optim., 41(2):563-582, February 2002.

[2] A. Berman, M. Neumann, and R.J. Stern. Nonnegative matrices in dynamic systems. Pure and applied mathematics. Wiley, 1989.

[3] Vahid S. Bokharaie, Oliver Mason, and Fabian Wirth. Stability and positivity of equilibria for subhomogeneous cooperative systems. Nonlinear Analysis: Theory, Methods \& Applications, 74(17):6416 6426, 2011.

[4] L. Farina and S. Rinaldi. Positive Linear Systems: Theory and Applications. A Wiley-Interscience publication. Wiley, 2000.

[5] H. R. Feyzmahdavian, T. Charalambous, and M. Johansson. Subhomogeneous positive monotone systems are insensitive to heterogeneous time-varying delays. In Proc. 21st International Symposium on Mathematical Theory of Networks and Systems (MTNS), 2014.

[6] H.R. Feyzmahdavian, T. Charalambous, and M. Johansson. Stability and performance of continuous-time power control in wireless networks. Automatic Control, IEEE Transactions on, 59(8):2012-2023, Aug 2014.

[7] G.J. Foschini and Z. Miljanic. A simple distributed autonomous power control algorithm and its convergence. Vehicular Technology, IEEE Transactions on, 42(4):641-646, Nov 1993.

[8] S. Haykin. Neural Networks: A Comprehensive Foundation. International edition. Prentice Hall, 1999.

[9] K.J. Hunt, D. Sbarbaro, R. Zbikowski, and P.J. Gawthrop. Neural networks for control systems - a survey. Automatica, 28(6):1083 1112, 1992.

[10] John A. Jacquez and Carl P. Simon. Qualitative theory of compartmental systems. SIAM Review, 35(1):43-79, 1993.

[11] Eugenius Kaszkurewicz and Amit Bhaya. Matrix diagonal stability in systems and computation. Birkhäuser, Boston, 2000.
[12] John Kennan. Uniqueness of positive fixed points for increasing concave functions on $\mathbb{R}^{n}$ : An elementary result. Review of Economic Dynamics, 4(4):893 - 899, 2001.

[13] A. Khanafer, T. Basar, and B. Gharesifard. Stability properties of infection diffusion dynamics over directed networks. In Decision and Control (CDC), 2014 IEEE 53rd Annual Conference on, pages 62156220, Dec 2014.

[14] U. Krause. Concave Perron-Frobenius theory and applications. Nonlinear Analysis: Theory, Methods \& Applications, 47(3):1457 - 1466, 2001.

[15] Patrick De Leenheer and Dirk Aeyels. Stabilization of positive linear systems. Systems \& Control Letters, 44(4):259 - 271, 2001.

[16] Chunguang Li, Luonan Chen, and K. Aihara. Stability of genetic networks with SUM regulatory logic: Lur'e system and LMI approach. Circuits and Systems I: Regular Papers, IEEE Transactions on, 53(11):2451-2458, Nov 2006.

[17] J. D. Murray. Mathematical biology. Springer-Verlag, Berlin, DEU, 2nd edition, 1993.

[18] Carlo Piccardi and Sergio Rinaldi. Remarks on excitability, stability and sign of equilibria in cooperative systems. Systems \& Control Letters, 46(3):153 - 163, 2002.

[19] D.D. Siljak. Large-Scale Dynamic Systems: Stability and Structure. North-Holland, 1978.

[20] Hal L. Smith. Monotone Dynamical Systems: An Introduction to the Theory of Competitive and Cooperative Systems, volume 41 of Mathematical Surveys and Monographs. AMS, Providence, RI, 1995.

21] E.D. Sontag. Structure and stability of certain chemical networks and applications to the kinetic proofreading model of t-cell receptor signal transduction. Automatic Control, IEEE Transactions on, 46(7):1028 -1047 , jul 2001.

[22] R.D. Yates. A framework for uplink power control in cellular radio systems. Selected Areas in Communications, IEEE Journal on, 13(7):1341-1347, Sep 1995.

[23] Huaguang Zhang, Zhanshan Wang, and Derong Liu. A comprehensive review of stability analysis of continuous-time recurrent neural networks. Neural Networks and Learning Systems, IEEE Transactions on, 25(7):1229-1262, July 2014. 